\title{
A TAUBERIAN RELATIVE OF THE LANDAU-INGHAM TAUBERIAN THEOREM
}

\author{
S. L. SEGAL 1
}

One of the earliest Tauberian theorems is Landau's $[4, \S 160]$.

TheOREM L. Suppose $F(x)$ is a real-valued function with $\sum_{1 \leq d \leq x} F(x / d)$ of bounded variation in every interval $[1, y]$ and

$$
\sum_{1 \leq d \leq x} F\left(\frac{x}{d}\right)=A x \log x+B x+O(w(x))
$$

where $w(x)$ is positive, and nondecreasing (from some point on), and such that

$$
\int_{1}^{\infty} \frac{w(t)}{t^{2}} d t
$$

converges. Then $F(x)=A x+o(x)$ as $x \rightarrow \infty$.

Landau's aim in proving this theorem was to deduce the prime number theorem (in an asymptotic form) from the result

$$
\sum_{n=1}^{\infty} \frac{\mu(n) \log n}{n}=-1,
$$

where $\mu(n)$ is the Möbius function. Later [5] Landau showed that only knowing

$$
\sum_{n=1}^{\infty} \frac{\mu(n)}{n}=0
$$

would suffice to prove Theorem $\mathrm{L}$, and this, together with his dissertation $[4, \S 156]$ comprised a proof that the prime number theorem was "elementarily equivalent" to $(* *)$.

Subsequently Ingham [3] proved, by using Wiener's Tauberian theory, the following result more general than Theorem $\mathrm{L}$.

Theorem I. Suppose

$$
\sum_{1 \leq d \leq x} F\left(\frac{x}{d}\right)=A x \log x+B x+o(x) .
$$

Presented to the Society, January 23, 1968 under the title Tauberian theorems for Dirichlet convolutions; received by the editors August 10, 1967 and, in revised form, October 25, 1967.

1 Partially supported by NSF Grant GP-5801. 
Then

(i) If $F(x)$ is positive and nondecreasing (from some point on), then $F(x)=A x+o(x)$. then

(ii) If $F(x)$ is bounded and integrable in every finite interval $[1, y]$,

$$
\int_{1}^{x} \frac{F(t)}{t^{2}} d t=A \log x+(B-A \gamma)+o(1)
$$

where $\gamma$ is Euler's constant.

In proving (i), no use is made of prime-number theory beyond the fact that the Riemann zeta-function $\zeta(s), s=\sigma+i t$, has no zeros on the line $\sigma=1$. In proving (ii), however, something more than (*) was required.

The asymptotic form of the prime number theorem follows from either of these theorems, on taking

$$
F(x)=\sum_{m=1}^{\infty} \sum_{p^{m} \leq x ; p \text { prime }} \log p
$$

and then applying standard arguments due to Tchebycheff.

However, it is easy to show (much simpler than the prime number theorem), and well known that if $\pi(x)=\sum_{p \leqslant x ; p \text { prime }} 1$, then

$$
\sum_{1 \leqq d \leqq x} \pi\left(\frac{x}{d}\right)=\sum_{p \leq x ; p \text { prime }}\left[\frac{x}{p}\right]=x \log \log x+K x+o(x)
$$

(e.g. [2, Theorem 430]) and this raises the question of what sort of conclusions can be drawn if $F(x)$ is a positive nondecreasing function satisfying, instead of (1),

$$
\sum_{1 \leq d \leq x} F\left(\frac{x}{d}\right)=A x \log \log x+B x+E(x),
$$

where $E(x)$ is an error term on which restrictions are to be placed. In particular, when can we conclude that $F(x) \sim A x / \log x$.

In this paper we prove a theorem of this kind. Although Ingham's method is not directly applicable to expressions of the form (3), the proof of the theorem stated below will depend on an appropriate use of both parts of Theorem I as well as an additional elementary Tauberian argument. The need for using Theorem I (ii) means that unfortunately we are using something stronger than (**) or even (*) and hence results substantially stronger than an asymptotic form of the prime number theorem. (See also the remarks after the proof of the theorem.) 
Throughout this paper, all functions are real-valued, all error terms are understood to be as the variable goes to $+\infty, \sum_{d \leq x}$ means a sum beginning at $1,[x]=\sum_{d_{s} x} 1,\{x\}=x-[x], p$ is a prime, $\pi(x)$ $=\sum_{p \leq x} 1, \gamma$ is Euler's constant, $K$ is the constant

$$
\gamma+\sum_{p} \log (1-1 / p)+1 / p
$$

All references to results of prime number theory not specifically attributed may be found in [4].

THEOREM. Suppose $a_{n} \geqq 0$ and let $F(x)=\sum_{n \leq x} a_{n}$. If

(4) $\sum_{d \leq x} F(x / d)=a x \log \log x+b x+c x / \log x+o(x / \log x)$, $a, b, c$ real constants, then $F(x)=\sum_{n \leq x} a_{n}=a x / \log x(1+o(1))$.

Proof. For convenience we define $F(x)=0$ for $x<1$. First observe that by (2) and (4)

$$
\sum_{d \leq x}\left(F\left(\frac{x}{d}\right)-a \pi(x / d)+a x / d\right)=a x \log x+(a \gamma+b-a K) x+o(x)
$$

and clearly $F(x)-a \pi(x)+a x$ is nondecreasing; hence by Theorem I (i) we have the preliminary estimate

$$
F(x)=o(x) .
$$

Now observe that by definition of $F(x)$,

$$
\begin{aligned}
\sum_{d \leq x} F\left(\frac{x}{d}\right) \log d & =\sum_{d \leq x} \log d \sum_{m \leq x / d} a_{m} \\
& =\sum_{d \leq x} a_{d}\left(\frac{x}{d} \log x / d-x / d+R(x / d)\right)
\end{aligned}
$$

where $R(x)=O(\log x)$, and since $a_{d} \geqq 0$ by hypothesis, we get by partial summation,

$$
\begin{aligned}
\sum_{d \leq x} F\left(\frac{x}{d}\right) & \log d \\
& =x \int_{1}^{x} \frac{1}{t} \sum_{d \leq t} \frac{a_{d}}{d} d t-x \sum_{d \leq x} \frac{a_{d}}{d}+O\left(\int_{1}^{x} \frac{F(t)}{t} d t\right) .
\end{aligned}
$$

But, using (5), and since, by partial summation,

$$
\sum_{d \leq x} \frac{a_{d}}{d}=\frac{F(x)}{x}+\int_{1}^{x} \frac{F(t)}{t^{2}} d t
$$

we get from (6), 


$$
\sum_{d \leq x} F\left(\frac{x}{d}\right) \log d=x \int_{1}^{x} \frac{d t}{t} \int_{1}^{t} \frac{F(u)}{u^{2}} d u+o(x) .
$$

We may also make a similar evaluation involving integrals of $F(x)$; in particular, on making a change of variables, and using (7), we have

$$
\begin{aligned}
\sum_{d \leq x} \log d \int_{1}^{x / d} \frac{F(t)}{t} d t & =\int_{1}^{x} \frac{1}{u} \sum_{d \leq u} \log d F(u / d) d u \\
& =\int_{1}^{x} d t \int_{1}^{t} \frac{d u}{u} \int_{1}^{u} \frac{F(v)}{v^{2}} d v+o(x) \\
& =\sum_{d \leq x} F(x / d) \log d-\int_{1}^{x} d t \int_{1}^{t} \frac{F(v)}{v^{2}} d v+o(x),
\end{aligned}
$$

on integrating by parts in the triply iterated integral. This last equality may be rewritten as

$$
\begin{aligned}
\sum_{d \leq x}\left(F\left(\frac{x}{d}\right) \log d\right. & \left.-\int_{1}^{x / d} \frac{F(t)}{t} d t \log d\right) \\
= & \int_{1}^{x} d t \int_{1}^{t} \frac{F(v)}{v^{2}} d v+o(x) .
\end{aligned}
$$

Combining (8) and (4) we have (since $\sum_{d_{5 x}} \int_{1}^{x / d}(F(t) / t) d t$ $\left.=\int_{1}^{x}(1 / v) \sum_{d \leq v} F(v / d) d v\right)$

$$
\begin{aligned}
& \sum_{d \leqq x}\left(F\left(\frac{x}{d}\right) \log \left(\frac{x}{d}\right)-\int_{1}^{x / d} \frac{F(t)}{t} d t \log \left(\frac{x}{d}\right)\right) \\
&=\sum_{d \leqq x} F\left(\frac{x}{d}\right) \log x-\int_{1}^{x} d t \int_{1}^{t} \frac{F(v)}{v^{2}} d v \\
&-(\log x) \int_{1}^{x} \frac{1}{u} \sum_{d \leq u} F(u / d) d u+o(x) \\
&= a x \log x \log \log x+b x \log x+c x+o(x) \\
&-\log x \int_{2}^{x} a \log \log u+b+\frac{c}{\log u}+o\left(\frac{1}{\log u}\right) d u \\
&-\int_{1}^{x} d t \int_{1}^{t} \frac{F(v)}{v^{2}} d v \\
&= a x+o(x)-\int_{1}^{x} d t \int_{1}^{t} \frac{F(v)}{v^{2}} d v .
\end{aligned}
$$

But by (5) again, 


$$
\begin{gathered}
\int_{1}^{x} d t \int_{1}^{t} \frac{F(v)}{v^{2}} d v=x \int_{1}^{x} \frac{F(v)}{v^{2}} d v-\int_{1}^{x} \frac{F(v)}{v} d v \\
=\int_{1}^{x} \frac{F(v)}{v}\left[\frac{x}{v}\right] d v+o(x)=\sum_{d \leq x} \int_{1}^{x / d} \frac{F(t)}{t} d t+o(x) \\
=\int_{1}^{x} \frac{1}{t} \sum_{d \leq t} F\left(\frac{t}{d}\right) d t+o(x) .
\end{gathered}
$$

Substituting (10) in (9) and using (4) again gives

$$
\begin{gathered}
\sum_{d \leqq x}\left(F\left(\frac{x}{d}\right) \log \left(\frac{x}{d}\right)-\int_{1}^{x / d} \frac{F(t)}{t} d t \log \left(\frac{x}{d}\right)\right) \\
=-a x \log \log x+(a-b) x+o(x) .
\end{gathered}
$$

And hence using (4) once more,

$$
\begin{gathered}
\sum_{d \leqq x}\left(F\left(\frac{x}{d}\right) \log \left(\frac{x}{d}\right)-\int_{1}^{x / d} \frac{F(t)}{t} d t \log \left(\frac{x}{d}\right)+F\left(\frac{x}{d}\right)\right) \\
=a x+o(x) .
\end{gathered}
$$

From Theorem I (ii) with $F(x)$ replaced by

$$
F(x) \log x-\int_{1}^{x} \frac{F(t)}{t} d t \log x+F(x),
$$

and with $A=0, B=a$ we now get

$$
\int_{1}^{x} \frac{F(u)(\log u+1)}{u^{2}} d u-\int_{1}^{x} \frac{\log u}{u^{2}} d u \int_{1}^{u} \frac{F(t)}{t} d t=a+o(1) .
$$

But using (5) still once more we have

$$
\begin{aligned}
-\int_{1}^{x} \frac{\log u}{u^{2}} d u \int_{1}^{u} \frac{F(t)}{t} d t & \\
& =\int_{1}^{x} \frac{1-\log u}{u^{2}} d u \int_{1}^{u} \frac{F(t)}{t} d t-\int_{1}^{x} \frac{d u}{u^{2}} \int_{1}^{u} \frac{F(t)}{t} d t \\
& =\frac{\log x}{x} \int_{1}^{x} \frac{F(t)}{t} d t-\int_{1}^{x} \frac{F(t)(\log t+1)}{t^{2}} d t+o(1)
\end{aligned}
$$

on integrating by parts in both integrals. Putting (14) in (13) thus gives

$$
\int_{1}^{x} \frac{F(t)}{t} d t=\frac{a x}{\log x}(1+o(1))
$$


An elementary and well-known Tauberian argument (reproduced e.g. in [1]) which we give for the sake of completeness, now completes the proof.

Since $F(x)$ is positive and nondecreasing $\left(a_{n} \geqq 0\right)$, we have from (15) for every $\epsilon>0$, for all sufficiently large $x$ (possibly depending on $\epsilon$ ),

$$
\begin{aligned}
F(x) \log (1+\epsilon) & \leqq \int_{x}^{x(1+\iota)} \frac{F(t)}{t} d t \\
& \leqq \frac{\left(a+\epsilon^{2}\right) x(1+\epsilon)}{\log x+\log (1+\epsilon)}-\frac{\left(a-\epsilon^{2}\right) x}{\log x}
\end{aligned}
$$

and hence

$$
\begin{aligned}
\frac{F(x) \log x}{x} & <\frac{\left(a+\epsilon^{2}\right)(1+\epsilon)-\left(a-\epsilon^{2}\right)}{\log (1+\epsilon)} \\
& =\frac{a \epsilon+2 \epsilon^{2}+\epsilon^{3}}{\log (1+\epsilon)}
\end{aligned}
$$

and so letting $\epsilon \rightarrow 0$ and $x \rightarrow \infty$

$$
\limsup _{x \rightarrow \infty} \frac{F(x) \log x}{x} \leqq a .
$$

Similarly one deduces from (15) that

$$
\liminf _{x \rightarrow \infty} \frac{F(x) \log x}{x} \geqq a
$$

and the theorem follows.

REMARKs. (i) It would be interesting if the proof of the theorem could be established using nothing more than $(* *)$ for a formula of type (4) for $\pi(x)$ follows immediately just from the asymptotic form of the prime number theorem: $\pi(x) \sim x / \log x$. Though this is well known, a proof does not appear to be in the literature and so is sketched here.

It is well known that from $\pi(x) \sim x / \log x$ one can deduce

$$
\sum_{n \leqq x} \frac{\Lambda(n)}{n}=\log x-\gamma+o(1)
$$

where $\Lambda(n)=\log p$ if $n=p^{m}, p$ a prime and 0 otherwise; and also 


$$
\sum_{p \leq x} \frac{1}{p}=\log \log x+K+o\left(\frac{1}{\log x}\right) .
$$

(For a method, see, e.g. Landau $[4, \S 55]$.) Hence we have from (17),

$$
\begin{aligned}
\sum_{d \leq x} \pi\left(\frac{x}{d}\right) & =\sum_{p \leq x}\left[\frac{x}{p}\right] \\
& =x \log \log x+K x+o\left(\frac{x}{\log x}\right)-\sum_{p \leq x}\left\{\frac{x}{p}\right\},
\end{aligned}
$$

where

$$
\begin{aligned}
\log x \sum_{p \leq x}\left\{\frac{x}{p}\right\}= & \sum_{p \leqq x} \log \left(\frac{x}{p}\right)\left\{\frac{x}{p}\right\}+\sum_{n \leq x} \Lambda(n)\left\{\frac{x}{n}\right\} \\
& -\sum_{p^{m} \leq x ; m \geq 2} \log p\left\{\frac{x}{p^{n}}\right\} .
\end{aligned}
$$

But here the third sum on the right is clearly $o(x)$, the first sum is positive and clearly $\leqq \sum_{p \leq x} \log (x / p)=o(x)$ by results of Tchebycheff, while for the second sum, by (16), and since

$$
\sum_{n \leq x} \Lambda(n)\left[\frac{x}{n}\right]=\sum_{n \leqq x} \sum_{d \mid n} \Lambda(d)=\sum_{n \leq x}^{-} \log ^{-} n,
$$

we have

$$
\begin{aligned}
\sum_{n \leq x} \Lambda(n)\left\{\frac{x}{n}\right\} & =x \log x-\gamma x+o(x)-x \log x+x+o(x) \\
& =(1-\gamma) x+o(x) .
\end{aligned}
$$

Hence putting these results in (19) and it in (18) gives

$$
\sum_{d \leqq x} \pi\left(\frac{x}{d}\right)=x \log \log x+K x+(\gamma-1) \frac{x}{\log x}+o\left(\frac{x}{\log x}\right) .
$$

(ii) Even if we showed that $F(x)(1+\log x)-\int_{1}^{x}(F(t) / t) d t \log x$ was a monotone function of $x$, the most we could conclude from Theorem I (i), using (5), would be

$$
F(x)-\int_{1}^{x} \frac{F(t)}{t} d t=o\left(\frac{x}{\log x}\right) .
$$

It is possible that (20) in conjunction with (4) might perhaps be 
used to prove the theorem and thus avoid the necessity of using Theorem I (ii), but I have not been able to do this.

\section{REFERENCES}

1. E. Grosswald, $A$ proof of the prime number theorem, Amer. Math. Monthly 71 (1964), 736-743.

2. G. H. Hardy and E. M. Wright, Introduction to the theory of numbers, Oxford Univ. Press, 1954.

3. A. E. Ingham, Some Tauberian theorems connected with the prime number theorem, J. London Math. Soc. 20 (1945), 171-180.

4. E. Landau, Handbuch der Lehre von der Verteilung der Primzahlen, 2nd ed., Chelsea, New York, 1953.

5. —— Über die Äquivalenz zweier Hauptsätze der analvtischen Zahlentheorie, Wiener Akademie Sitzungsberichte 20 (1911), 973-988.

UNIVERSITY OF ROCHESTER 